\title{
ÉDITORIAL
}

\section{Dix ans et toujours en train d'apprendre à marcher}

\author{
Jim Ducharme, MD
}

ENGLISH VERSION ON PAGE 127

$\mathrm{C}$ 'était en avril 1999 qu'un nouveau journal naissait, ponctué de pleurs et de hurlements, comme c'est le cas pour tous les bébés en santé. Ses parents n'arrivaient pas à décider quel nom lui donner, mais ils ont fini par s'entendre pour l'appeler le Journal canadien de la médecine d'urgence (JCMU). Ils étaient si fiers, comme en témoigne leur première déclaration : « La première chose que vous constatez lorsque vous devenez rédacteur d'un nouveau journal, c'est qu'il n'y a aucun article à publier. C'est une bonne chose, car cela vous permet de déterminer, sans influence indue, la direction que le journal devrait prendre $»^{1}$.

Garth Dickinson, premier rédacteur associé principal du $J C M U$, déclarait : « Un autre journal de médecine d'urgence? Il y en a déjà des tonnes! Certains vous parviennent sans que vous y soyez abonné. Vous souvenez-vous du CAEP Review au début des années 80? Il n'y avait pas que le titre qui était nul. ${ }^{2} \mathrm{Ah}$, les parents! Ils sont si fiers qu'ils ne peuvent cacher leur enthousiasme!

Et c'est ainsi que tout a commencé. Comment avonsnous pu prendre notre envol alors que la plupart des lecteurs misaient contre notre survie? Après tout, on avait échoué auparavant, comme l'avait dit Garth Dickinson de manière si laconique. La réponse est aussi simple qu'incroyable : c'est tout simplement le fait d'une pure volonté. Grant Innes, à titre de premier rédacteur en chef, a concentré toute sa volonté pour que JCMU s'épanouisse, consacrant d'innombrables heures chaque semaine à son siège social — le sous-sol de sa maison — travaillant fébrilement jusqu'au petit matin aux côtés d'une directrice de la rédaction tout aussi dévouée, sa femme.

La deuxième raison pour laquelle nous avons réussi : nous n'avions pas d'orgueil. Avez-vous une idée du temps que nous avons consacré à mendier (et à proférer des menaces) pour qu'on nous présente des articles de toute nature pour un journal inconnu et non indexé qui ressurgit tous les 2 mois?

La troisième raison de notre succès : une croyance en la qualité de l'enseignement de la médecine d'urgence dans ce pays, conjuguée à une ignorance absolue de la quantité de travail bénévole nécessaire. Grant a réuni nos premiers rédacteurs associés (Ian G. Stiell, Jacques S. Lee, Jim Thompson, Laurie J. Morrison, Julie Spence, John Ross, Michael J. Bullard, David J. Rhine, Anthony S. Taylor, Cheri Nijssen-Jordan, Bruce Minnes, Al Huber, Tim Allen, Isser Dubinsky, Jason R. Frank, Kirk Hollohan et Jeffrey L. Arnold) et, avec leur énergie et leurs connaissances, a permis de tirer de la farine d'un sac de son. Nous leur sommes profondément reconnaissants du temps, de l'engagement et de l'énergie qu'ils ont donnés à ce journal.

À mesure que nous grandissions, nous sommes devenus la source de normes internationalement reconnues en médecine d'urgence comme le Système canadien d'information de gestion des départements d'urgence (SIGDU) ${ }^{3}$ et l'Échelle canadienne de triage et de gravité (ÉTG) pour les services d'urgence ${ }^{4}$. Nous avons publié de nouvelles données sur le SRAS à un moment où ce syndrome captivait le monde entier ${ }^{5}$, tout cela même si nous n'étions pas encore indexés par la National Library of Medicine des États-Unis et reconnus officiellement comme revue spécialisée.

Notre croissance a été douloureuse et ardue, mais le jeu en a valu la chandelle. La contribution des bénévoles est encore colossale et largement méconnue. Il est difficile de réduire la charge de chaque examinateur ou rédacteur, étant donné le nombre limité de médecins universitaires disponibles au Canada. Et pourtant, ils sont si nombreux à

Rédacteur en chef, JCMU; professeur clinicien de médecine, Université McMaster University, Hamilton (Ontario)

Les opinions exprimées dans les éditoriaux sont celles des auteurs; elles ne reflètent pas nécessairement celles du JCMU ou de l'Association canadienne des médecins d'urgence.

CJEM 2009;11(2):129-30 
donner de leur temps. Comment, en tant que deuxième rédacteur en chef du JCMU, puis-je les remercier suffisamment? Peut-être que la meilleure façon de le faire serait de leur communiquer les commentaires positifs que nous recevons régulièrement au sujet de la qualité du journal et comment, pour tant de gens, le JCMU est une revue médicale canadienne pertinente. Une autre façon serait d'être témoins du nombre grandissant d'articles que nous recevons de l'étranger et de leur qualité croissante. Il n'y a pas si longtemps, Grant, Garth et moi passions des heures à réécrire des articles pour nous assurer d'avoir suffisamment d'articles acceptables pour publication. Aujourd'hui, nous examinons des articles de qualité et, s'il y a lieu, nous pouvons nous permettre de demander aux auteurs de faire mieux.

«Sommes-nous arrivés au bout de nos peines? » La réponse est « Non ». En fait, je ne sais pas si l'on n'y arrivera jamais. Ma foi, je ne suis même pas sûr de savoir où se situe le «bout de nos peines »! Nous sommes maintenant indexés, comme l'annonçait allègrement Grant Innes en $2007^{6}$. Nous publions des articles importants sur le plan politique ${ }^{7}$ ainsi que des lignes directrices cliniques de pointe ${ }^{8}$. Nous n'avons certes pas le facteur d'impact du Lancet, de Science, ou même d'Academic Emergency Medicine, mais vous savez quoi? Cela m'importe peu que nous atteignions un tel succès. Mon seul désir est que chaque numéro continue d'avoir une incidence sur les médecins d'urgence au Canada et soit à la fois « divertissant » et intéressant pour nos lecteurs. N'est-ce pas ce que tout enfant de 10 ans serait fier d'avoir accompli?

Je vais donc revenir en arrière et aller jouer à la « tague » pour voir ce qui va se passer. Qui sait ... peut-être qu'un jour Kirby battra Marth à Super Smash Bros Brawl. Et que le « geek » sera invité à danser! Mais, à 10 ans, les meilleures années du JCMU sont devant lui. Bon sang, n'est-ce pas ce que la vie a à offrir à cet âge-là? Rien n'est impossible et l'avenir est devant vous!

Conflit d'intérêts : Aucun déclaré.

Mots clés : médecine d'urgence, publication, lignes directrices, énoncé de position

\section{Références bibliographiques}

1. Innes G. Méditations sur un nouveau journal [éditorial]. JCMU 1999;1:15-16.

2. Dickinson G. Un nouveau journal de médecine d'urgence? [éditorial] JCMU 1999;1:18.

3. Grafstein E, Unger B, Bullard M, et al; Canadian Emergency Department Information System (CEDIS) Working Group. Canadian Emergency Department Information System (CEDIS) Presenting Complaint List (Version 1.0). CJEM 2003;5:27-34.

4. Beveridge R, Clarke B, Janes L, et collab. L'échelle canadienne de triage et de gravité pour les départements d'urgence : Guide d'implantation. JCMU 1999;1(3 suppl):S2-28.

5. Wong WN, Sec ACH, Lau RFL, et al. Accuracy of clinical diagnosis versus the World Health Organization case definition of the Amoy Garden SARS cohort. CJEM 2003;5:384-91.

6. Innes G. Ruminations of a (newly indexed) journal. CJEM 2007;9:5-8.

7. Snider CE, Ovens H, Drummond A, et collab. Énoncé de position de l'ACMU sur le contrôle des armes à feu. JCMU 2009; 11:73-83.

8. Green RS, Djogovic D, Gray S, et collab. Lignes directrices de l'Association canadienne des médecins d'urgence sur le sepsis : la prise en charge optimale du sepsis grave dans les départements d'urgence canadiens. JCMU 2008;10:553-71.

Correspondance : cjem@caep.ca 